\title{
Continuous Models Fail to Capture Details of Reentry in Fibrotic Myocardium
}

\author{
Tanmay A Gokhale, Eli Medvescek, Craig S Henriquez \\ Duke University, Durham, United States
}

\begin{abstract}
Cardiac arrhythmias have traditionally been simulated using continuous models that assume tissue homogeneity and use a relatively large spatial discretization. However, it is believed the tissue fibrosis and collagen deposition, which occur on a micron-level scale, are critical factors in arrhythmogenesis, and it remains unclear how well continuous models are able to accurately capture complex conduction behaviors such as reentry in fibrotic tissue. The objective of this study was to compare reentrant behavior in discrete microstructural models of fibrosis and in two types of equivalent continuous models, a homogenous model and a hybrid continuous model with distinct heterogeneities.

In the discrete model, increasing levels of tissue fibrosis lead to substantial increases in reentrant cycle length which are inadequately reflected in the homogenous and hybrid continuous models. These cycle length increases appear to be due primarily to increases in tip path length and to altered restitution behavior, and suggest that it is critical to consider the discrete effects of fibrosis on conduction when studying arrhythmogenesis in fibrotic myocardium.
\end{abstract}

\section{Introduction}

Computational models of cardiac conduction can play an important role in understanding the mechanisms of and developing treatments for fibrosis-induced cardiac arrhythmias. However, most studies investigating the arrhythmogenic role of cardiac fibrosis have modelled the myocardium as a locally homogenous continuum with increased conduction anisotropy in areas of fibrosis. While these continuum methods recreate simple planar conduction behaviour and allow for increased computational efficiency, it remains unclear how effectively they are able to model complex conduction patterns such as reentry.

\subsection{Discrete versus continuous models}

In 1981, Spach et al. [1] were the first to show that while cardiac conduction is continuous on the macroscopic scale, microscopic conduction is discontinuous and affected by the discrete cellular structure of the tissue. Since that time, many studies have used discrete microstructural cardiac models that incorporate individual cellular geometry [2][4]. Despite the discrete nature of cardiac conduction, many other studies have relied on continuum models that represent averaged electrical properties of cardiac tissue because these models decrease computational load and allow for large-scale simulations, including those of whole-heart conduction. While these continuous models, where the electrical properties are assigned to match macroscopic conduction properties and anisotropy, have been shown to reproduce simple conduction behaviours with high fidelity, it remains unclear if such models can account for the effects of fibrosis on the microscale.

\subsection{Modeling of fibrotic myocardium}

The most histologically accurate representation of fibrosis is as space-occupying features separating cells in discrete tissue models. Because collagenous septa are nonconductive, they can be more easily represented as the decoupling of transverse cellular connections [5].

In continuous computational models, fibrosis is traditionally incorporated by decreasing conductivity values to reproduce experimentally observed conduction slowing and conduction anisotropy. While this approach reproduces the macroscopic conduction behavior, it does not capture the microscopic effects of fibrosis that could be implicated in arrhythmogenesis [6]. The more recent approach by Costa et al [7] of decoupling FEM elements to reproduce the effects of fibrosis is analogous to the approach of Spach et al. in the discrete model, and has shown promise in reproducing microscale conduction. However, it remains unclear how any of these approaches perform in the setting of reentry spiral wave behavior.

In this manuscript, we elicit and compare spiral wave behavior in discrete models as well as in two types of equivalent continuous models to understand whether continuous computational models are sufficient to capture the details of reentry.

\section{Methods}




\subsection{Tissue models}

Two dimensional monodomain microstructural models of anisotropic cardiac monolayers that incorporate discrete, uniformly distributed gap junctions were randomly generated using previously described methods [8]. Tissues were $2 \mathrm{~cm} \times 2 \mathrm{~cm}$, with a spatial discretization of $20 \mathrm{um}$, and individual myocytes were an average of 100 um in length and 20 um in width. An intracellular resistivity of $150 \mathrm{ohm}^{*} \mathrm{~cm}$ and a gap junction conductance of 2.5 uS were used. Collagen septa of variable length (mean $=800$ micron) were inserted, parallel to cardiac fiber orientation, to disrupt between $0 \%$ and $30 \%$ of the transverse coupling between myocytes. $70 \%$ of the remaining combined plicate transverse junctions were decoupled to replicate adult tissue conductivity. Five distinct microstructural models with random septa placement and transverse decoupling were constructed for each level of fibrosis $(0 \%, 10 \%, 20 \%$, and $30 \%)$.

Equivalent homogenous continuous models with a spatial discretization of 100 um were produced for each fibrosis density by adjusting the tissue conductivities to match the conduction velocities (CVs) of transverse and longitudinal conduction observed in the discrete model. Hybrid continuous models were created by inserting longitudinally oriented decoupling septa of mean length 800 micron into a homogenous continuous model to disrupt between $0 \%$ and $30 \%$ of transverse coupling between nodes. The electrical conductivities between the tissue nodes were then adjusted such that the combination of decoupling septa and tissue conductivity resulted in conduction velocities equivalent to each discrete model.

\subsection{Simulation of conduction}

In each microstructural and continuous model, longitudinal and transverse conduction velocities were measured by initiating a plane wave along the left or top boundary of the tissue. Activation time was measured as the time to half-maximal voltage and action potential duration was measured as time to $80 \%$ repolarization. Conduction velocities were calculated by performing a linear regression on activation times.

Reentry was induced by application of cross-field S1S2 stimulation. A planar wave was initiated by a stimulation along the left boundary of the tissue (S1). When the planar wavefront had crossed $70 \%$ of the longitudinal tissue distance $(1.4 \mathrm{~cm})$. a second stimulus (S2) was applied in the top left region of the tissue by an electrode $800 \mathrm{um}$ in length and $1.6 \mathrm{~cm}$ in width. A stable spiral wave was allowed to form for at least three full revolutions before the cycle length was measured at several points on the tissue. Tip trajectories were tracked by finding the intersection of the wavefront $(\mathrm{dV} / \mathrm{dt}=0)$ and the waveback (a fixed potential of $-75 \mathrm{mV}$ ).
Restitution was measured by pacing the tissue in the transverse direction at $2 \mathrm{~Hz}$ for 5 pulses, followed by application of a premature transverse stimulus of the same amplitude. The velocity of conduction and action potential duration resulting from the premature stimulus were recorded, and the interval between the final pacing stimulus and the premature stimulus was decreased until the premature stimulus no longer elicited a propagating wave front.

\subsection{Numerical methods}

All simulations were performed using the Cardiowave software package [9] (available online at cardiowave.duke.edu). The Bondarenko membrane model of the mouse myocyte [10] was used in all simulations because of its relatively short action potential duration.

\subsection{Analytical methods}

All data is presented as mean +/- standard deviation unless otherwise specified. A two-way ANOVA with posthoc pairwise Tukey HSD was used to identify significant differences between models at different fibrosis densities.

\section{Results and discussion}

\subsection{Planar stimulation}

Conduction velocity was measured in the microstructural model after applying planar stimulation along the top or left edge of the tissue. As the degree of fibrosis was increased, conduction velocity in the longitudinal direction decreased only slightly from $41.02 \pm$ $0.03 \mathrm{~cm} / \mathrm{s}$ with no fibrosis to $39.58 \pm 0.07 \mathrm{~cm} / \mathrm{s}$ with $30 \%$ fibrosis ( $\mathrm{n}=5$ for each case).

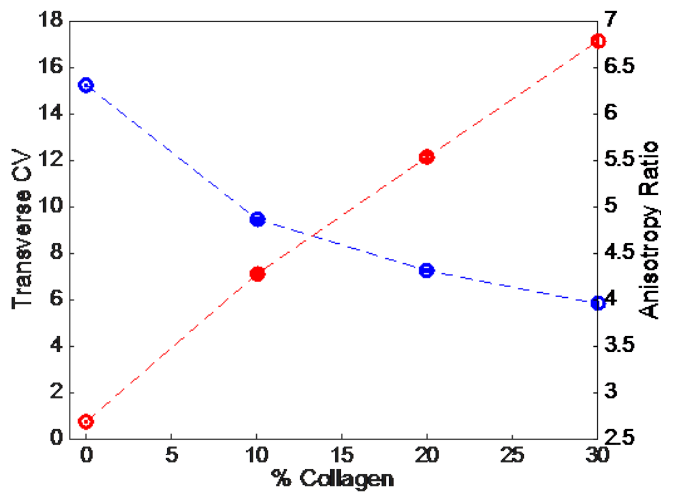

Figure 1. Conduction slowing in the discrete model due to increased fibrosis. ( $\mathrm{n}=5$ tissues per case). ${ }^{*} \mathrm{p}<0.05$

Transverse conduction velocity slowed substantially with increased fibrosis, from $15.24 \pm 0.01 \mathrm{~cm} / \mathrm{s}$ to $5.83 \pm$ $0.04 \mathrm{~cm} / \mathrm{s}$ as fibrosis was increased from $0 \%$ to $30 \%$, 
resulting in an increase in anisotropy ratio from 2.6:1 to 6.8:1 (Figure 1).

\subsection{Spiral wave activity}

Spiral waves were induced in microstructural models and in the equivalent continuous and hybrid models by application of cross-field stimulation. Action potential traces were recorded at several points in the domain away from the spiral tip. Comparison of voltage traces reveals that the continuous model closely matches the behaviour of the discrete model in the control, non-fibrotic case, but a substantial difference in traces is noted at higher fibrosis densities (Figure 2).

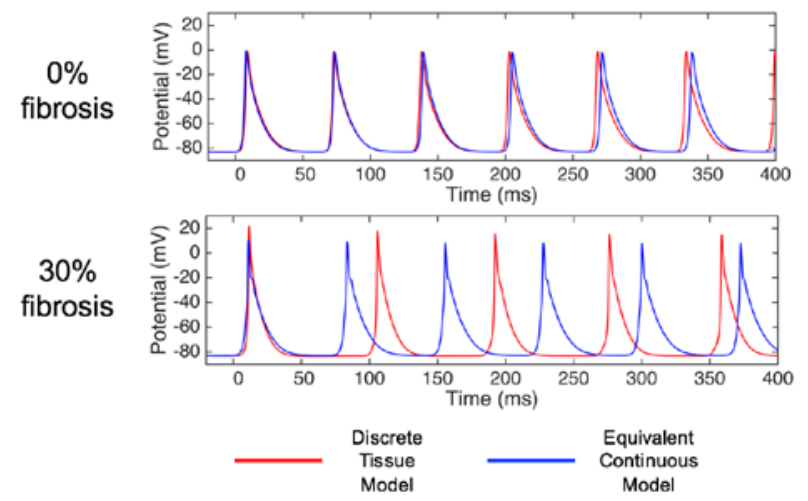

Figure 2. Voltage traces measured in discrete models (red) and in equivalent continuous models (blue) reveal a substantial difference in behavior in high fibrosis cases (bottom panel) but not in the control case (top panel)

In the discrete model, increasing fibrosis led to a prolongation of mean cycle length from $65.0 \mathrm{~ms}$ in nonfibrotic tissue to $86.6 \mathrm{~ms}$ in $30 \%$ fibrosis, an increase of 33.2 percent (Figure 3). In addition, as fibrosis was increased, there was an increase in the variability of cycle length between tissues of equal fibrosis.

Spiral waves were similarly induced in the continuous tissue models with equivalent planar conduction velocities. Cycle length in the non-fibrotic continuous model was similar to that observed in the microstructural model (66.8 ms continuous vs $65.0 \mathrm{~ms}$ discrete). However, at higher degrees of fibrosis, the continuous tissues showed minimal increase in cycle length, with a maximal increase to 72.3 ms (8.1\% increase) in 30\% fibrosis-equivalent tissue.

The hybrid model showed moderate increases in cycle length due to increasing fibrosis, with a maximum cycle length of $80.6 \mathrm{~ms}$ in $30 \%$ fibrosis-equivalent tissue (a $20.6 \%$ increase from the cycle length of 66.8 in the nonfibrotic tissue model). Thus, the hybrid model is able to more accurately simulate reentrant behavior compared to the homogenous continuous model, but falls short of matching the behavior of the discrete model.

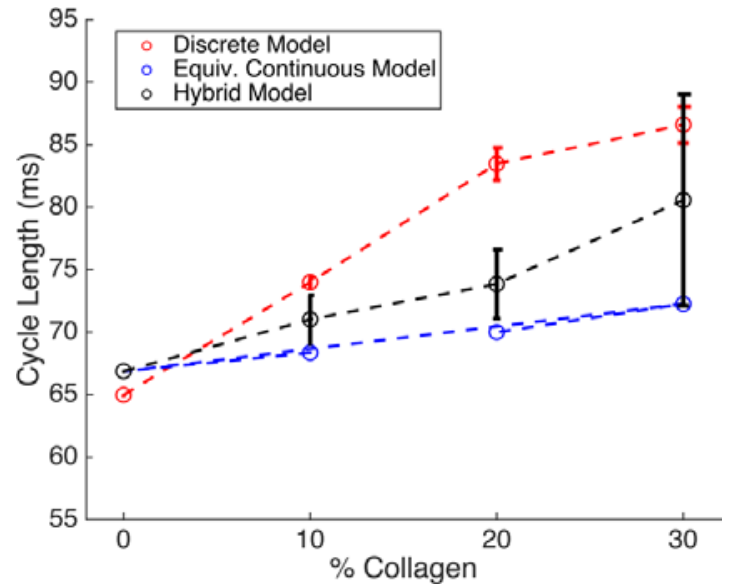

Figure 3. Increases in the cycle length of reentry seen in microstructural/discrete models (red) are not seen in the equivalent continuous models (blue) or the hybrid model (black) ( $\mathrm{n}=5$ tissue models per case)

\subsection{Factors affecting cycle length}

To understand what factors lead to the longer cycle in the discrete model that are not fully captured in the continuous and hybrid models, the impact of tip trajectory and restitution behaviour were explored.

Analysis of tip trajectory in the control case showed that discrete and both continuous models exhibited similar tip trajectories around an elliptical unexcitable core of approximately $2 \mathrm{~mm}$ in length and $0.8 \mathrm{~mm}$ in width. In the $20 \%$ fibrosis case, the tip trajectory in the homogenous continuous model remained relatively unchanged, with an elliptical trajectory around an unexcitable core, and the minimal increase in cycle length was due to slowed transverse conduction. However, in the discrete model, the tip trajectory was much longer and thinner (approximately $8 \mathrm{~mm}$ in length), with no unexcitable core, suggesting an anatomical rather than functional form of reentry. The hybrid model exhibited tip trajectories qualitatively similar to those of the discrete model, but with a less dramatic increase in longitudinal tip travel (approximately $6 \mathrm{~mm}$ in length).

Restitution behavior in the discrete and the homogenous continuous models with $20 \%$ fibrosis was examined using a standard S1-S2 pacing protocol. Because the homogenous continuous model was constructed to replicate discrete model conduction velocities at $1 \mathrm{~Hz}$ pacing, the conduction velocities are equivalent in the two models at longer diastolic intervals (above $200 \mathrm{~ms}$ ). However, as the diastolic interval is decreased, the discrete model exhibits a substantial slowing of conduction velocity not seen in the homogenous continuous model (Figure 4), with statistically significant differences seen at diastolic intervals of $100 \mathrm{~ms}$ and shorter $(\mathrm{p}<0.05)$. This behavior 
suggests that while equivalent continuous models are able to replicate planar conduction properties at a single diastolic interval, alteration of the diastolic interval alone causes the continuous model to deviate from discrete model behavior.

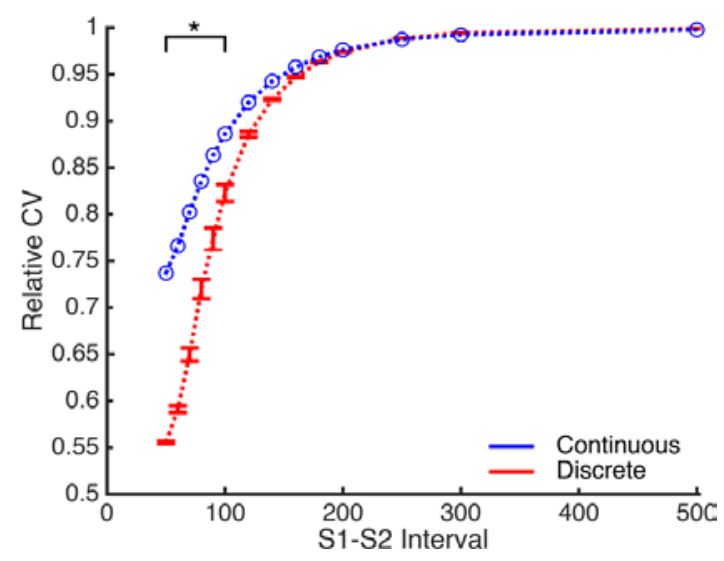

Figure 4: Conduction velocity restitution behaviour differs substantially between the homogenous continuous model (blue) and the discrete model (red) at shortened diastolic intervals. ${ }^{*} \mathrm{p}<0.05$

The impact of collagenous septa length was also examined. When mean septa length in the discrete model was reduced to 200 um, no difference in cycle length was observed between the discrete and the equivalent homogenous continuous model, and tip trajectory remained elliptical around an unexcitable core at fibrosis densities up to $50 \%$.

\section{Conclusions}

These results indicate that local discontinuities due to fibrosis appear to have a significant impact on complex conduction behaviour, and neither homogenous continuous models nor hybrid models are able to fully capture this effect for sub-millimeter discontinuities. However, the hybrid model appears to be promising in capturing discrete model behaviour, and alterations in hybrid model construction may improve accuracy.

It remains unclear what method of modelling fibrosis is optimal in continuous models. Different types of tissue fibrosis will likely impose different limitations on what models are able to recapitulate. We believe it is critical to consider and validate methodologies of incorporating fibrosis in computational tissue models before these models can be used to gain mechanistic understanding of and devise treatments for clinical arrhythmia.

\section{Acknowledgements}

This work was supported by National Institutes of
Health grants R01HL093711 (to C.H) and 5T32GM007171 to the Duke Medical Scientist Training Program (T.G).

\section{References}

[1] M. S. Spach, W. T. Miller, D. B. Geselowitz, R. C. Barr, J. M. Kootsey, and E. a Johnson, "The discontinuous nature of propagation in normal canine cardiac muscle. Evidence for recurrent discontinuities of intracellular resistance that affect the membrane currents.," Circ. Res., vol. 48, pp. 3954, 1981.

[2] M. S. Spach and J. P. Boineau, "Microfibrosis produces electrical load variations due to loss of side-to-side cell connections: a major mechanism of structural heart disease arrhythmias.," Pacing Clin. Electrophysiol., vol. 20, no. 2 Pt 2, pp. 397-413, Feb. 1997.

[3] M. S. Spach, J. F. Heidlage, R. C. Barr, and P. C. Dolber, "Cell size and communication: role in structural and electrical development and remodeling of the heart.," Heart Rhythm, vol. 1, no. 4, pp. 500-15, Oct. 2004.

[4] J. Stinstra, R. MacLeod, and C. Henriquez, "Incorporating histology into a 3D microscopic computer model of myocardium to study propagation at a cellular level.," Ann. Biomed. Eng., vol. 38, no. 4, pp. 1399-414, Apr. 2010.

[5] M. S. Spach, J. F. Heidlage, P. C. Dolber, and R. C. Barr, "Mechanism of origin of conduction disturbances in aging human atrial bundles: experimental and model study.," Heart Rhythm, vol. 4, no. 2, pp. 175-85, Feb. 2007.

[6] M. L. Hubbard and C. S. Henriquez, "A microstructural model of reentry arising from focal breakthrough at sites of source-load mismatch in a central region of slow conduction.," Am. J. Physiol. Heart Circ. Physiol., vol. 306, no. 9, pp. H1341-52, May 2014.

[7] C. M. Costa, F. O. Campos, A. J. Prassl, R. W. dos Santos, D. Sánchez-Quintana, H. Ahammer, E. Hofer, and G. Plank, "An efficient finite element approach for modeling fibrotic clefts in the heart.," IEEE Trans. Biomed. Eng., vol. 61, no. 3, pp. 900-10, Mar. 2014.

[8] V. Jacquemet and C. S. Henriquez, "Loading effect of fibroblast-myocyte coupling on resting potential, impulse propagation, and repolarization: insights from a microstructure model," AJP Hear. Circ. Physiol., vol. 294, no. 5, pp. H2040-H2052, Mar. 2008.

[9] J. Pormann, "A modular simulation system for the bidomain equations,” Duke University, 1999.

[10] V. E. Bondarenko, "Computer model of action potential of mouse ventricular myocytes,” AJP Hear. Circ. Physiol., vol. 287, no. 3, pp. H1378-H1403, 2004.

Address for correspondence:

Tanmay Gokhale

CIEMAS 1427

Box 90281

Durham, NC 27708-0281

tanmay.gokhale@duke.edu 